\title{
The Management of Post-Keratoplasty Glaucoma by Trabeculectomy
}

\author{
A. M. E. GILVARRY, C. M. KIRKNESS, A. D. McG. STEELE, N. S. C. RICE, \\ L. A. FICKER \\ London
}

\begin{abstract}
Summary
The results of 35 consecutive trabeculectomies in eyes developing medically uncontrollable glaucoma following penetrating keratoplasty are presented, with a mean follow-up of 3 years from the time of drainage surgery. Five eyes remained phakic until trabeculectomy was performed. Additional medical therapy was necessary to control the intraocular pressure in 32 eyes, which therefore were considered to have failed to be controlled by trabeculectomy and $90 \%$ of these failed within 6 months of filtration surgery. Despite additional medical therapy, in 17 eyes, further drainage surgery was required and $90 \%$ of this surgery took place within the first 14 months. Adverse prognostic factors were multiple grafts and synechial closure of the drainage angle.
\end{abstract}

Since the establishment of penetrating keratoplasty as a realistic surgical procedure producing good visual results, glaucoma has been a well recognised complication. Although the improvement of microsurgical techniques has eliminated some of the earlier forms of glaucoma associated with leaking wounds and synechiae to the graft hose interface, glaucoma remains a serious and sight-threatening problem ${ }^{1-4}$. As more cases of severe anterior segment disorganisation become technically operable, there is more likelihood of raised intraocular pressure following surgery ${ }^{5}$.

Aged, aphakic and traumatised eyes are all at considerable risk of developing this form of glaucoma as are eyes which have perforated, and also regrafted eyes ${ }^{6}$.

Medical therapy for post-keratoplasty glaucoma (PKPG) relies heavily on the use of carbonic anhydrase inhibitors. Nearly all the topically applied medications have adverse reactions which may preclude or limit their use in individual cases. Once the intraocular pressure (IOP) has failed to be controlled on maximum tolerable medical therapy, or progressive optic nerve damage occurs, the surgical options for control of the IOP are limited.

Both cyclodialysis and cyclocryotherapy have been advocated. Casey ${ }^{7}$ showed that cyclodialysis carried a very poor prognosis for maintenance of normal IOP and graft clarity. Cyclocryotherapy has been described as useful for the control of post-keratoplasty glaucoma but the follow-up was short $^{8}$. Recently, Kirkness et al demonstrated that the long-term results of this form of cycloablation were very poor both from the point of view of survival of graft clarity and the maintenance of normal pressure ${ }^{9}$ with none of 13 patients maintaining both a clear graft and an IOP less than $21 \mathrm{mmHg}$. More advanced cases

From: Corneal Clinic, Moorfields Eye Hospital, City Road, London.

Correspondence to: Colin M Kirkness, FRCS, FCOphth, Department of Clinical Ophthalmology, Institute of Ophthalmology, Moorfields Eye Hospital, City Road, London EC1V 2PD. 
were shown to respond well to the use of silicone drainage tubing although the rate of complication was high ${ }^{9}$. Laser trabeculoplasty has also been shown to be effective in reducing the pressure in aphakic grafts, but in most cases the reduction in pressure was not sufficient to permit withdrawal of any of the antiglaucoma medication during an average follow-up of 20 months. ${ }^{10}$

In many cases, however, it may be felt that trabeculectomy is the procedure of first choice when medical control is lost. Trabeculectomy is a well established surgical procedure, indicated for many forms of glaucoma with a recognisably high degree of success.

There are circumstances, however, $e g$ when there is considerable conjunctival scarring, when a filtration bleb may be difficult to establish and the trabeculectomy fails ${ }^{11,12}$. It was our clinical impression that PKPG did not respond as well to conventional trabeculectomy as might be hoped. In an effort to determine the success of trabeculectomy in PKPG and to highlight the risk factors, we reviewed all patients undergoing trabeculectomy for post-keratoplasty glaucoma under the care of the surgeons of the Corneal Clinic at Moorfields Eye Hospital, London.

\section{Patients and Methods}

Patients who had undergone trabeculectomy following penetrating keratoplasty were identified from a database of all corneal clinic patients. Those patients still attending the clinic were reviewed and three patients who had discontinued attendance had their case notes reviewed.

The indications for trabeculectomy were failure to control intraocular pressure on maximum tolerable medical therapy. In addition, three patients had had one previous drainage procedure in that eye. This included two Scheie Procedures, one 19 years prior to keratoplasty and one 9 years prior to keratoplasty. One other patient had had multiple intraocular procedures for congenital glaucoma including one drainage procedure, probably a Scheie Procedure.

The trabeculectomy was carried out in the standard fashion usually using a limbus-based conjunctival flap.

The procedure was deemed successful if the intraocular pressure was kept below $21 \mathrm{mmHg}$ without the use of additional antiglaucoma medication. The term 'partial success' was used to describe those cases where additional antiglaucoma treatment was required to bring the IOP below $21 \mathrm{mmHg}$. When even maximum tolerable therapy was insufficient to control the IOP below $21 \mathrm{mmHg}$, the case was described as a failure. Most cases in this latter group underwent further glaucoma surgery. Where progressive field loss or optic disc cupping occurred in the presence of apparently normal IOPs, the trabeculectomy was also said to have failed. Survival curves were plotted according to the methods of Kaplan \& Meier ${ }^{13}$.

\section{Results}

Over a 12 year period to December 1988 , there were 35 trabeculectomies for PKPG. There were 18 males and 17 females with ages ranging from 24 to 84 yrs (mean 58, median $64)$. In these 35 eyes, at the time of trabeculectomy 25 eyes had had one penetrating keratoplasty and 10 had had two or more corneal grafts. Only two eyes remain phakic, but five were phakic when the trabeculectomy was performed. All patients who were aphakic at the time of keratoplasty had an open sky anterior vitrectomy with a vitreous cutting instrument used during the procedure. The relationship between cataract surgery and the keratoplasty is shown in Table I. The original indication for keratoplasty is shown in Table II.

Eighty-three per cent of the eyes in this series had significant, documented conjunctival scarring prior to trabeculectomy being performed. In $46 \%$ of cases the drainage angle was closed by peripheral anterior synechiae. In some cases these obliterated the entire anterior chamber. In others the angle was essentially open although there were a few fine peripheral anterior synechiae in almost every case.

The time interval between the keratoplasty and trabeculectomy is shown in Figure 1. It ranged from two days to 3.75 years with a mean of seven months. Ninety per cent of the trabeculectomies were performed within the first 14 months of graft surgery.

The success of trabeculectomy is shown in 
Table I The distribution of eyes in this series according to the type of cataract surgery

Still phakic

ICCE before PK

ICCE with IOL before PK

ECCE before PK

ECCE with IOL before PK

Combined PK and ECCE

Combined PK and ICCE

Combined PK, ECCE and IOL

ECCE and IOL after PK

ECCE and PK

Aphakia due to trauma after PK

Table II The indications for penetrating keratoplasty of the eyes in this series

\begin{tabular}{lc}
\hline $\begin{array}{l}\text { Indications for } \\
\text { penetrating keratoplasty }\end{array}$ & Number \\
\hline Bullous keratopathy & 10 \\
Herpes simplex keratitis & 6 \\
Microbial keratitis & 6 \\
Perforation & 3 \\
Trauma & 2 \\
Miscellaneous & 8 \\
\hline
\end{tabular}

Figure 2. Glaucoma surgery failed from within one week to one year but $90 \%$ had failed to be controlled followed surgery alone within 25 weeks and additional medical therapy had to be instituted. At the time of this review only three eyes are maintained with normal intraocular pressure without the need for additional antiglaucoma medication and of these only two of the grafts are clear.

The additional antiglaucoma medication necessary to control IOP varied from timolol drops to multiple therapy including timolol, pilocarpine, phospholine iodide and acetazolamide $250 \mathrm{mg}$ four times a day. (Fig 3). In 17 eyes, even additional and maximally tolerable therapy was insufficient to control the glaucoma adequately and further surgery was necessary. This absolute failure of the original trabeculectomy is shown in Fig 4. The majority of those failing and requiring further surgery did so within the first year following the original trabeculectomy. The additional surgery performed (Fig 3B), was most commonly the insertion of a silicone drainage tube.

Only one serious peri-operative complication was encountered-delayed supra- choroidal haemorrhage which was successfully drained but the patient's vision went from $6 / 36$ to counting fingers. Visual acuity was maintained in $86 \%$ of cases. Eyes with closed angle PKPG were significantly more likely to fail after trabeculectomy than those in which the angle was open $p=0.005$ by $\chi^{2}$ testing.

Of the 25 first grafts, nine were successfully or partially-successfully controlled by trabeculectomy and of the ten further grafts only two were controlled partially-successfully. This difference is significant at $\mathrm{p}=0.025$ by $\chi^{2}$.

In the group of successful and partially successful control (18 eyes), 16 grafts remained clear whereas in the group failing (17 eyes) only seven grafts remained clear, this difference is significant at $p=0.005$ by $\chi^{2}$ testing. No relationship could be seen between the nature of lens surgery and the success of trabeculectomy; or between the length of time between trabeculectomy and the original keratoplasty, and the success of the glaucoma surgery.

\section{Discussion}

The failure of trabeculectomy to control intraocular pressure below $21 \mathrm{mmHg}$ in certain eyes has long been recognised, in the young, in blacks and in eyes with secondary glaucoma ${ }^{14,17}$. Not only is an already scarred conjunctiva a handicap but the aqueous from eyes that have undergone intraocular surgery shows increased chemo-attractant activity towards fibroblasts from Tenon's capsule. ${ }^{12}$ In our series of eyes with PKPG, $86 \%$ were

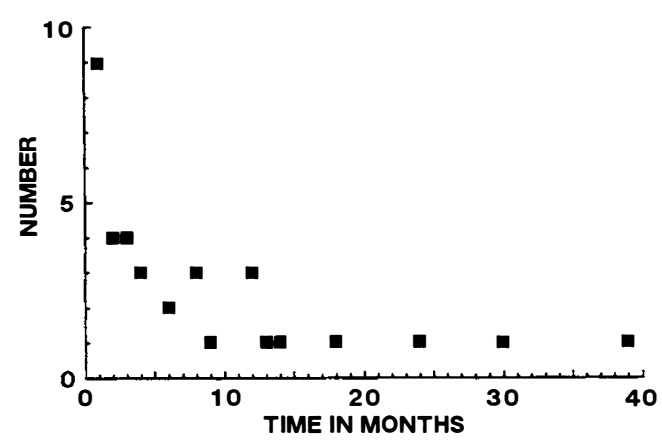

Fig. 1. Graph showing the interval between penetrating keratoplasty and trabeculectomy. 


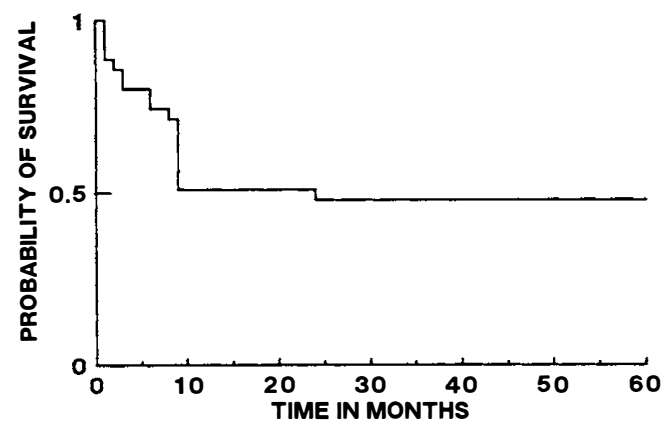

Fig. 2. Survival curve of the trabeculectomy. Survival is defined as the maintenance of $I O P<21 \mathrm{mmHg}$ without any additional antiglaucoma therapy.

aphakic at the time of trabeculectomy, $83 \%$ had scarred conjunctivae (none of the aphakic eyes had had cataract surgery through a clear corneal incision) and all had had at least one previous intraocular procedure. These adverse factors were compounded by the preponderance of eyes in which the indication for keratoplasty was inflammatory disease or severe anterior segment disorganisation where damage to the angle might be expected. It is, perhaps, not surprising that these eyes should have been found to have a relatively poor result from drainage by trabeculectomy. In the 16 eyes where the angle was closed by extensive anterior synechiae, there was a significantly worse prognosis compared to the rest of the group as far as IOP control was concerned.

In 17 eyes, even additional medical therapy was insufficient to control the glaucoma adequately and further surgery was necessary. The majority of those failing and requiring further surgery did so within the first year following trabeculectomy. Nearly half the patients, however, were controllable on medical therapy though, in some cases, this amounted to a great deal of both topical and systemic medication. In this group, successful or partially successful (18 eyes), 16 grafts remained clear whereas in the group failing (17 eyes) only seven grafts remained clear. The difference is significant at $p=0.005$. Of the 25 first grafts, nine were successfully or partially-successfully controlled by trabeculectomy and of the ten further grafts only two were controlled partially-successfully. This difference is significant at $p=0.025$. No relationship could be seen between the nature of lens surgery and the success of trabeculectomy, nor between the length of time between trabeculectomy and the original keratoplasty and the success of the glaucoma surgery. This latter fact may suggest that the degree of ongoing post-operative inflammation was not germane to the success of filtration surgery.

\section{Trabeculectomy.}

Comparable studies are difficult to find but in one series ${ }^{17}$, Gross found only four out of 15 aphakic patients had adequate control of IOP by trabeculectomy nine months following surgery using criteria for success broadly similar to our definition of partial success. On this basis, in our series $70 \%$ were adequately controlled at nine months and by two years $50 \%$ were still adequately controlled. Bellows ${ }^{18}$, again using similar criteria for adequate control, found a two year survival of $65 \%$ and a three year survival of $50 \%$ (ie by extrapolation from his published data to produce survival curves). Huer ${ }^{19}$ using somewhat more liberal criteria for success, nevertheless, found a $50 \%$ failure rate among aphakic trabeculectomies aged 50 years or over. Of these, $90 \%$ had failed within nine months but it was not possible to extrapolate from his data to obtain survival curves. The difficulty of comparing data in different series emphasises the need for such series to demonstrate success of the techniques described in a dynamic

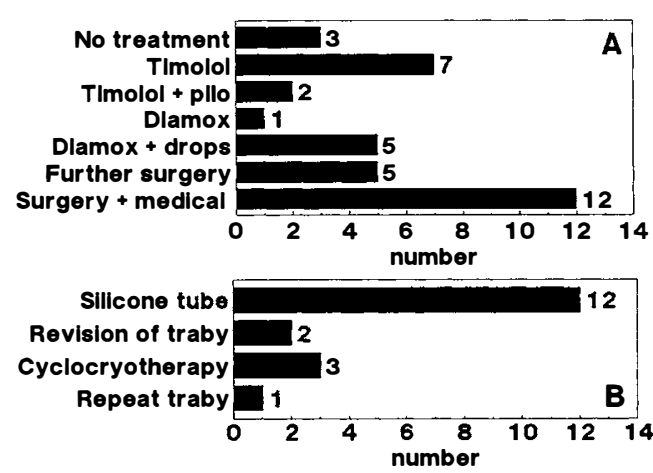

Fig. 3. The distribution of various treatment regimes by individual patients at the time of review.

$A$. Distribution of medical and surgical treatment.

$B$. Subset of the 'further surgery and surgery + medical' groups in $A$ showing the type of surgery which was subsequently undertaken. 


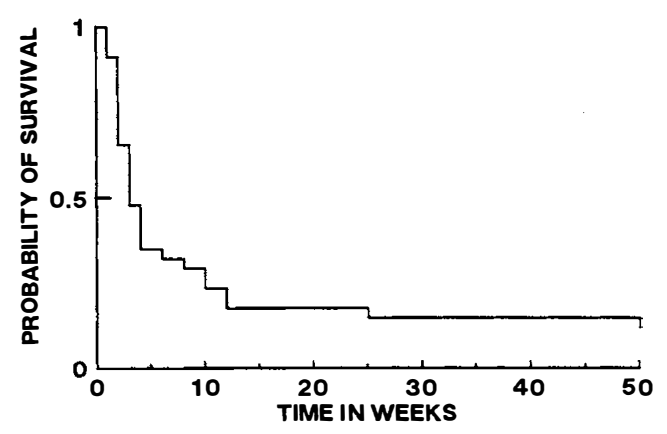

Fig. 4. The survival curve of the trabeculectomy with use of additional medical therapy. Failure is defined as the inability of the trabeculectomy and maximum tolerable additional antiglaucoma medical therapy to control the $I O P<21 \mathrm{mmHg}$.

fashion ie by the use of survival curves. Considering these series of trabeculectomy in aphakia, our figures for PKPG are broadly similar in the success of filtration surgery and are encouraging in view of the fact that PKPG may be considered the more difficult to manage surgically. Van Mete ${ }^{\prime} s^{10}$ results for LTP in open or partially open angle PKPG show that the IOP may be reduced to within normal limits while medical therapy is continued. This indicates that LTP could be considered prior to trabeculectomy once the IOP becomes unstable.

The use of 5-fluorouracil has not been described in trabeculectomy for PKPG but its use is under widespread investigation where bleb fibrosis may be anticipated. Given by injection in $5 \mathrm{mg}$ doses, subconjunctivally, it may limit fibroblastic activity. It is, however known to produce toxic reactions in the conjunctiva and corneal epithelium resulting in larger and persistent epithelial defects, predisposing to microbial keratitis ${ }^{20,21}$. This is especially likely to occur when the drug is in direct contact with corneal epithelium. In view of this, its use in PKPG may be limited since longstanding epithelial defects may result in intrastromal scarring and reduced acuity. There may be a place, however, for cautious trial of this drug given by a fine bore needle, to prevent leakage, subconjunctivally, high in the superior fornix.

\section{Cyclocryotherapy.}

In a series using cyclocryotherapy to treat aphakic glaucoma ${ }^{22}$, approximately $70 \%$ of patients had pressure reduction below $21 \mathrm{mmHg}$ but the data are not presented in a way that survival rates may be determined. Forty per cent of the eyes, however, lost substantial amounts of vision as a consequence of the treatment. The authors do not discuss the precise nature of the visual loss. Binder ${ }^{8}$ found cyclocryotherapy useful in PKPG after a relatively short follow-up, but even so a number of serious complications were encountered, some proceeding to loss of light perception. Kirkness ${ }^{9}$ in a small series, demonstrated that no eye out of 13 reported maintained both a clear graft and normal intraocular pressure.

\section{Silicone tubes}

At first sight, silicone tubes would seem to offer at least as good a prognosis for maintenance of normal IOP but their insertion is associated with considerably more complications both in the early and late post-operative periods. ${ }^{9}$ While these may be managed successfully four out of 20 eyes lost vision in the post-operative period reported. Other authors have reported an even higher incidence of complications and graft failures albeit using a different surgical technique and post-operative drug regime ${ }^{23}$. In addition, complications such as incarceration of vitreous or iris in the tube which necessitates further intraocular intervention may prejudice survival of the graft either from endothelial damage or from graft reactions. It is noteworthy that there were few serious complications in the series undergoing trabeculectomy although it could be argued that graft failure was predisposed by further intraocular surgery. From a larger series in preparation (personal communication) it appears that graft survival is at least $80 \%$, two years after intraocular surgery.

Our figures indicate that trabeculectomy carries only a limited prognosis for maintenance of normal IOP after penetrating keratoplasty without the need for additional medical therapy. In view of the risks associated with alternative procedures, it may yet be preferable as the procedure of first choice. Second or further grafts and those eyes with closed angle PKPG, however, have signifi- 
cantly reduced chance of successful trabeculectomy. Should further surgical intervention be indicated, our experience leads us to suggest that a silicone drainage tube should be inserted in a two-stage fashion. Cyclocryotherapy should be reserved for those eyes without functional vision.

\section{References}

${ }^{1}$ Irvine AR, and Kaufman HE: Intraocular pressure following keratoplasty. Am J Ophthalmol 1969, 68: $835-8$.

${ }^{2}$ Olson RJ and Kaufman HE: Prognostic factors of intraocular pressure after aphakic keratoplasty. Am J Ophthalmol 1975, 86: 510-15.

${ }^{3}$ Lass JH, and Pavan-langston D: Timolol therapy in secondary angle closure post penetrating keratoplasty. Ophthalmology 1979, 86: 51-9.

${ }^{4}$ Cohen EJ, Kenyon K, Dohlman CH: Iridoplasty for the prevention of post-keratoplasty angle closure and glaucoma. Ophthalmic Surg 1982, 13: 994-6.

${ }^{5}$ Kirkness CM, Steele ADMcG, Rice NSC: Penetrating keratoplasty in the management of suppurative keratitis. Developments in Ophthalmology (Karger, Basel) 1989, 18: 172-5.

${ }^{6}$ Kirkness CM and Moshegov C: Post-keratoplasty glaucoma. Eye 1988, 2Suppl: S19-S26.

${ }^{7}$ Casey TA and Gibbs D: Complications in corneal grafting. Trans Ophthalmol Soc UK 1972, 92: 517-30.

${ }^{8}$ Binder PS, Abel RA, Kaufman HE: Cyclocryotherapy for glaucoma after penetrating keratoplasty. Am J Ophthalmol 1975, 75: 489-92.

${ }^{9}$ Kirkness CM, Ling Y, Rice NSC: The use of silicone drainage tubing to control post-keratoplasty glaucoma. Eye 1988, 2: 583-90.

${ }^{10}$ Van Meter WS, Allen RC, Waring III GO, Stulting $\mathrm{RD}$ : Laser trabeculoplasty for glaucoma in aphakic and pseudophakic eyes after penetrating keratoplasty. Arch Ophthalmol 1988, 106: 185-8.

${ }^{11}$ Hitchings RA and Grierson I: Clinico-pathological correlation in eyes with failed fistulising surgery. Arch Ophthalmol 1988, 103: 84-8.

12 Joseph JP, Miller MH, Hitchings RA: Wound healing as a successful barrier to successful filtration surgery. Eye 1988, 2 Suppl: S113-S23.

${ }^{13}$ Kaplan E and Meier P: Nonparametric estimation from incomplete observations. J Am Statist Ass 1958, 53: 202-3.

${ }^{14}$ Gressel MG, Heuer DK, Parrish RK: Trabeculectomy in young patients. Ophthalmology 1984, 91: $1242-6$.

${ }^{15}$ Singleton BJ, Distler JA, Baker BH: Filtration surgery in black patients - early results in a West Indian population. Ophthal Surg 1987, 18: 195-9.

${ }^{16}$ Miller MH, Joseph NH, Wishart PK, Hitchings RA: Lack of beneficial effect of intensive topical steroids and beta radiation of eyes undergoing repeat trabeculectomy. Ophthal Surg 1987, 18: 508-12.

${ }^{17}$ Gross RL, Feldman RM, Spaeth GL et al.: Surgical therapy of chronic glaucoma in aphakia and pseudophakia. Ophthalmology 1988, 95: 1195201.

${ }^{18}$ Bellows AR and Johnstone A: Surgical management chronic glaucoma in aphakia. Ophthalmology 1983, 90: 807-13.

${ }^{19}$ Huer DK, Gressel MG, Parrish RK et al.: Trabeculectomy in aphakic eyes. Ophthalmology 1984, 91: 1045-51.

${ }^{20}$ Shapiro MS, Thoft RA, Friend J et al.: 5-fluorouracil toxicity to the ocular surface epithelium. Invest Ophthal Vis Sci. 1985, 26: 580-3.

${ }^{21}$ Knapp A, Hueur DK, Stern GA et al.: Serious corneal complications of glaucoma filtering surgery with post-operative 5-fluorouracil. $\mathrm{Am} J$ Opthalmol 1987, 103: 183-7.

${ }^{22}$ Caprioli J, Strang SL, Spaeth GL et al.: Cyclocryotherapy in the treatment of advanced glaucoma. Ophthalmology 1985, 92: 947-54.

${ }^{23}$ Mcdonnell PJ, Robin JB, Schanzlin DJ et al.: Molteno implant for the control of glaucoma in eyes after penetrating keratoplasty. Ophthalmology, 1988, 95: 364-9. 